\title{
A 4-D Hyperchaotic Memristive Dynamical System
}

\author{
Dimitrios Prousalis ${ }^{1, a}$, Christos Volos ${ }^{1, b}$, loannis Stouboulos ${ }^{1, c}$, and Ioannis Kyprianidis ${ }^{1, d}$ \\ ${ }^{1}$ Department of Physics, Aristotle University of Thessaloniki, Thessaloniki, GR-54124, Greece
}

\begin{abstract}
A new 4-D memristive system is presented in this paper. The peculiarity of the model is that it displays a line of equilibrium points and it has hyperchaotic dynamics in a particular range of the parameters space. The behavior of the proposed system is investigated through numerical simulations, by using phase portraits, Lyapunov exponents and Poincaré sections. Furthermore, we have designed and implemented an electronic circuit to verify the feasibility of the proposed system.
\end{abstract}

\section{Introduction}

Chua introduced the missing fourth circuit element, the memristor, in 1971 according to the theory of electrical circuits combining the charge $q$ and the flux $\phi$ [1]. A general concept of memristive systems was developed by Chua and Kang [2]. However until 2008 when Strukov et al at Hewlett-Packard Laboratories [3] reported a solid-state implementation of the memristor, the research on memristors was not broad enough. In 2009, Ventra et al [4] proposed other "mem-elements", such as memcapacitor memcapacitor and meminductor. Since then, many potential applications about memristors have been found based on their properties. Non-volatile memory applications, crossbar latches as transistor replacements or augmentors, analog computation and circuit applications, as well as memristor-based neural networks are some of them. Also, the design of memristor-based chaotic oscillators, by replacing the nonlinear part of chaotic dynamical systems with memristors has been introduced [5]-[7]. So, research on memristor-based chaotic systems becomes a focal research topic in both the technological and the application domain [8]-[16].

Furthermore, in recent years a new classification of chaotic dynamics has been introduced. According to this, there are two types of attractors: self-excited attractors and hidden attractors. A self-excited attractor has a basin of attraction that is excited from unstable equilibria. In contrast, hidden attractor cannot be found by using a numerical method in which a trajectory starts from a point on the unstable manifold in the neighbourhood of an unstable equilibrium [17]. Studying hyperchaotic systems with hidden attractors is still an open research topic. Motivated by complex dynamical behaviors of hyperchaotic systems, noticeable characteristics of memristor and unknown fea-

\footnotetext{
ae-mail: dprou@physics.auth.gr

be-mail: volos@physics.auth.gr

ce-mail: stouboulos@physics.auth.gr

de-mail: imkypr@auth.gr
}

tures of hidden attractors, a memristive hyperchaotic system with a line of equilibrium points is proposed in this paper.

This research work is organized as follows. In Section 2 a 3-D four-wing chaotic system, the model of the memristive system, as well as the new hyperchaotic system are presented. In Section 3 presents the simulation results of the memristive system. In Section 4 the circuit realization of the system is described in detail and Section 5 concludes this work with a symmary of the main results.

\section{The New 4-D Hyperchaotic Memristive System}

\subsection{The 3-D Four-Wing Chaotic System}

In $2015 \mathrm{Li}$ et al [18] announced a 3-D four-wing system which is described by the equations:

$$
\begin{aligned}
& \dot{x}=a x+y+y z \\
& \dot{y}=-x z+y z \\
& \dot{z}=-z-m x y+b
\end{aligned}
$$

The system of differential equations (1) is a chaotic nonlinear system with four quadratic nonlinearities, three linear terms and especially with none in the second equation.

It has been shown by Li et al [18] that the 3-D system (1) displays the following features.

- It generates a four-wing chaotic attractor with fractal structure for some choices of the parameters.

- In a selected parameter region, it displays robust chaos with periodic Arnold tongues, and the strange attractor varies among a symmetric pair of single-wing attractors, a double-wing butterfly attractor, and a four-wing attractor.

- There is a hidden parameter in the coefficient of a quadratic nonlinearity in the system, whereby an obvious constant term controls the amplitude of all variables. 


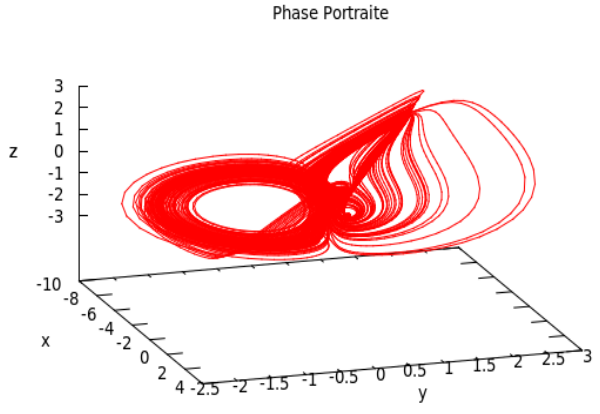

Figure 1. Phase space in $x-y-z$, for $a=0.4, b=3, c=0.1$, $d=6, e=1$.

The attractor resembles two groups of butterfly shapes, which is different from other reported four-wing systems. The Figure 1 shows that system's (1) four wings are built in different way.

\subsection{Model of the Memristive System}

As it is mentioned, Chua and Kang introduced the memristive system by generalizing the original definition of a memristor [2]. A memristive system can be described as:

$$
\begin{aligned}
& \dot{w}_{m}=F\left(w_{m}, u_{m}, t\right), \\
& f_{m}=G\left(w_{m}, u_{m}, t\right) u_{m}
\end{aligned}
$$

where $w_{m}, f_{m}$ and $u_{m}$ denote the state of memristive system, output and input, respectively. The function $G$ is a continuous and $n$-dimensional scalar function and $F$ is a vector function. So in this work the following form of a memristive system has been used:

$$
\begin{aligned}
& \dot{w}_{m}=u_{m}, \\
& f_{m}=\left(1+0.25 w_{m}^{2}-0.002 w_{m}^{4}\right) u_{m} .
\end{aligned}
$$

In order to investigate the behavior of the memristive system an external bipolar period signal $u_{m}$ is applied. The form of $u_{m}$ is:

$$
u_{m}=A \sin (2 \pi f t)
$$

where $A$ is the amplitude and $f$ is the frequency. From the first equation of the system (3) we can find $w_{m}$ :

$$
w_{m}=w_{m}(0)+\frac{A}{2 \pi f t}(1-\cos (2 \pi f t))
$$

where $w_{m}(0)=\int_{-\infty}^{0} u_{m}(\tau) d \tau$ is the initial condition of the internal state $w_{m}$.

Substituting (4) and (5) into (3), it is easy to derive the output of the memristive system. Therefore, the output $y_{m}$ depends on the frequency and amplitude of the applied input stimulus. Figure 2 shows the hysteresis loops of the memristive system (3) when it is driven by a periodic signal (4) with different frequencies. Obviously, the proposed memristive system exhibits a pinched hysteresis loop in the input-output plane.

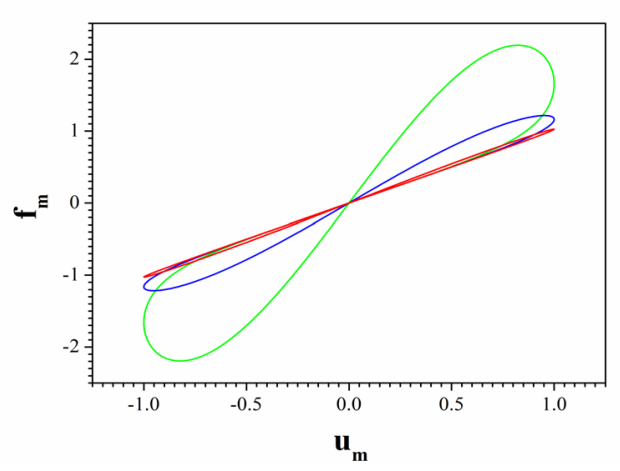

Figure 2. Hysteresis loops of the proposed memristive system driven by a sinusoidal stimulus with $A=1$ and $w_{0}=0$, for $f=0.1$ (green line), $f=0.2$ (blue line), $f=0.5$ (red line).

\subsection{The New Hyperchaotic Memristive System}

Finally, according to the aforementioned 3-D chaotic system and memristive system, the following 4-D memristive system is presented.

$$
\begin{aligned}
& \dot{x}=a x+b y+y z \\
& \dot{y}=-x z+y z-c f \\
& \dot{z}=-z-d x y+e \\
& \dot{w}=y
\end{aligned}
$$

where $y=u_{m}, w=w_{m}$ and $f=f_{m}$.

The 4-D memristive system (6) for every $a, b, c, d, e$ has a line of equilibrium points $E\left(0,0, e, w_{m}\right)$. As a result, this memristive hyperchaotic system can be considered as a dynamical system with hidden attractors because it is impossible to verify the chaotic attractor by choosing an arbitrary initial condition in the vicinity of the unstable equilibria [18].

The Four-Wing hyperchaotic attractor in the $(\mathrm{x}, \mathrm{y}, \mathrm{z})$ phase space, for $a=0.5, b=3, c=0.1, d=6, e=1$ is depicted in Fig. 3.

By using the Wolf et al [19] algorithm the Lyapunov exponents have been calculated as: $L_{1}=0.35891, L_{2}=$ $0.00239, L_{3}=0$ and $L_{4}=-4.1826$. So, there are two positive Lyapunov exponents as a result the system is hyperchaotic. In addition the Kaplan-Yorke dimension of the system is found as:

$$
D_{K Y}=3+\frac{L_{1}+L_{2}+L_{3}}{\left|L_{4}\right|}=3.0864
$$

As it is shown in Fig. 3 the new 4-D memristive system has the same "butterfly" shape.

\section{Simulation Results}

In order to study the behavior of the new system, usual assets of the theory of dynamical systems such as phase portaits, Poincaré maps, bifurcation diagrams and and diagram of Lyapunov exponents.

The bifurcation diagram of $y$ versus $\alpha$, for $b=3$, $c=0.1, d=6, e=1$, is presented in Fig. 4. Also 


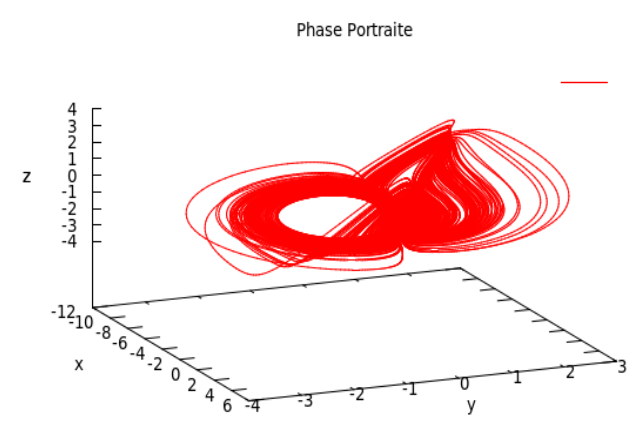

Figure 3. Hyperchaotic attractor in $(x, y, z)$ phase space, for $a=$ $0.5, b=3, c=0.1, d=6, e=1$.

the diagram of system's (6) Lyapunov exponents versus $\alpha$ is depicted in Fig. 4. This diagram confirms system's dynamical behavior as it is presented in the respective bifurcation diagram. At the Lyapunov diagram (Fig. 5) the fourth Lyapunov exponent is ignored because it takes negative values far from the zero value.

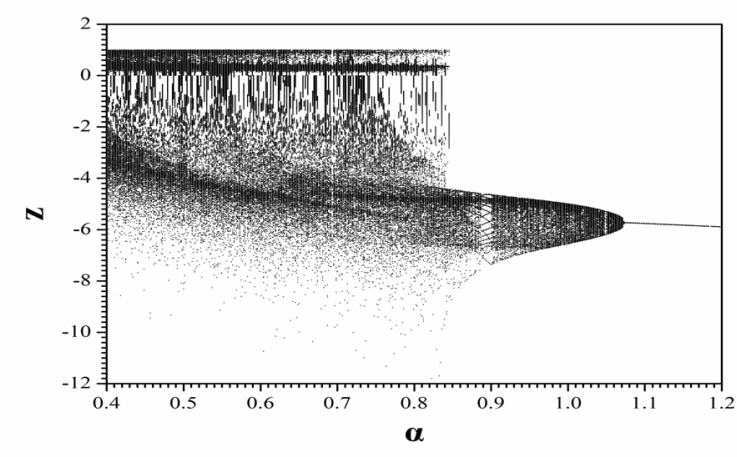

Figure 4. The bifurcation diagram of $y$ versus $\alpha$ for $b=3, c=$ $0.1, d=6, e=1$.

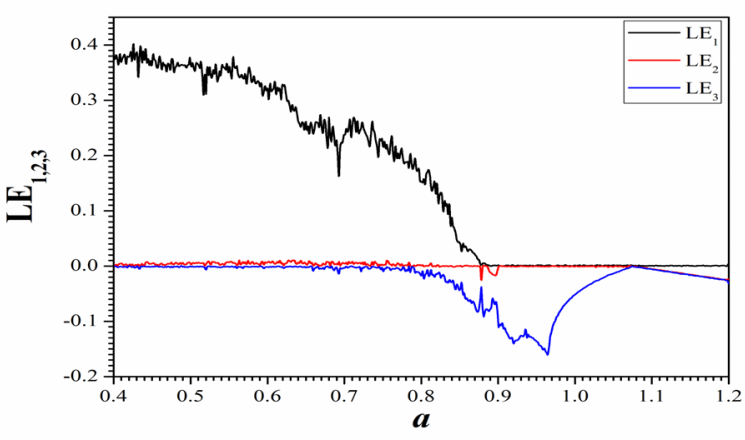

Figure 5. The Lyapunov Diagram of $y$ versus $\alpha$ for $b=3, c=$ $0.1, d=6, e=1$.
In more details, system (6) presents the following dynamical behavior:

- a region of periodic behavior for $a>1.07$ (Fig. 6). .

- a region of quasi-periodic behavior for $0.91<a<1.07$ (Fig. 7).

- a region of periodic behavior for $0.89<a<0.91$.

- a region of chaotic behavior for $0.85<a<0.89$ (Fig. 8).

- a region of hyperchaotic behavior for $0.4<a<0.85$ (Fig. 9).

Especially, the hyperchaotic behavior is shown in the Lyapunov diagram of Fig. 5 in the region where two Lyapunov Exponents become positive and one zero.

\section{Circuit Realization of the Memristive Hyperchaotic System}

The electronic circuit, which emulates the mathematical model of the proposed memristive hyperchaotic system (6), is presented in this section, in order to show its feasibility. The designed circuit, which is built by using offthe-shelf electronic components, is an effective way for discovering dynamics of the theoretical model practically. Circuital design, especially of hyperchaotic systems plays an important role on the field of nonlinear science due to its applications in many fields, such as in secure communication, signal processing, random bit generator, or path planning for autonomous mobile robot etc [20]-[24]. In addition, circuital implementation of chaotic/hyperchaotic systems is also provide an effective approach for investigating dynamics of such theoretical models [25], [26]. For example, hyperchaotic attractors can be observed on the oscilloscope easily or experimental bifurcation diagrams can be obtained by varying the values of variable resistors [27], [28].

Figure 10 depicts the schematic of the circuit. The main circuit that realizes the system (6), has four integrators (U1 - U4) and two inverting amplifiers (U5, U6), which are implemented with the operational amplifier TL084, as well as three signals multipliers (U8 - U10) by using the ana$\log$ multiplier AD633. Also, the circuital realization of the memristor's function $f$ of Eq. (2), is also depicted in Fig. 10 . Indeed the sub-circuit of the proposed memristive system only emulates the memristive function because there is not any commercial off-the-shelf memristive device in the market yet. So, the memristor's function is realized by using an inverting adder amplifier (U7) and three signals multipliers (U11 - U13). By applying Kirchhoff's circuit laws, the corresponding circuital equations of the designed circuit can be written as:

$$
\begin{aligned}
& \dot{x}=\frac{1}{R \cdot C}\left[\frac{R}{R_{a}} x+\frac{R}{R_{b}} y+\frac{R}{10 V \cdot R_{1}} y \cdot z\right] \\
& \dot{y}=\frac{1}{R \cdot C}\left[-\frac{R}{10 V \cdot R_{1}} x \cdot z+\frac{R}{10 V \cdot R_{1}} y \cdot z-\frac{R}{R_{c}} f\right] \\
& \dot{z}=\frac{1}{R \cdot C}\left[-z-\frac{R}{10 V \cdot R_{d}} x \cdot y+V_{e}\right] \\
& \dot{w}=\frac{1}{R \cdot C} y
\end{aligned}
$$

where

$$
f=\left[\frac{R}{10 V \cdot R_{1}} V_{f}+\frac{R}{(10 V)^{2} \cdot R_{2}} w^{2}-\frac{R}{(10 V)^{4} \cdot R_{3}} w^{4}\right] y
$$




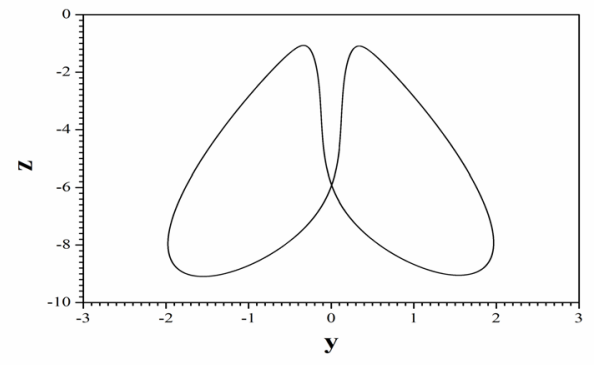

(a)

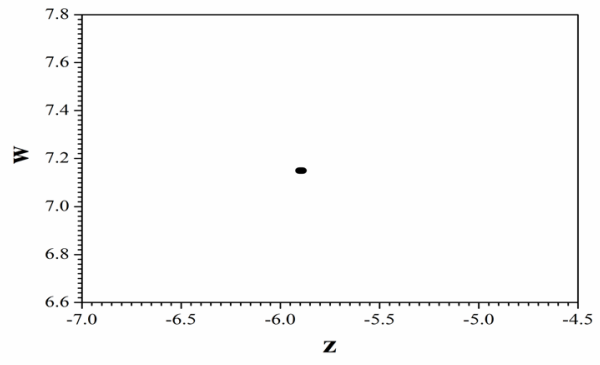

(b)

Figure 6. (a) Phase portrait and (b) Poincaré map, for $\alpha=1.2, b=3, c=0.1, d=6, e=1$.

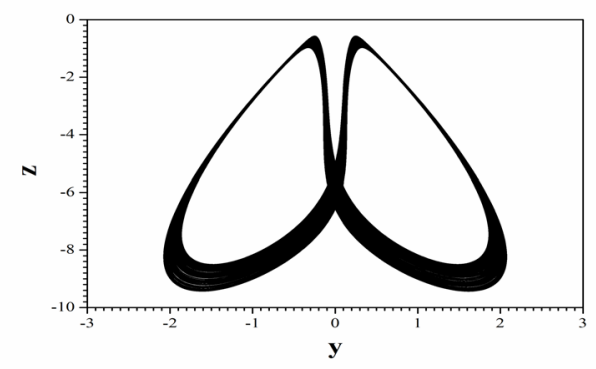

(a)

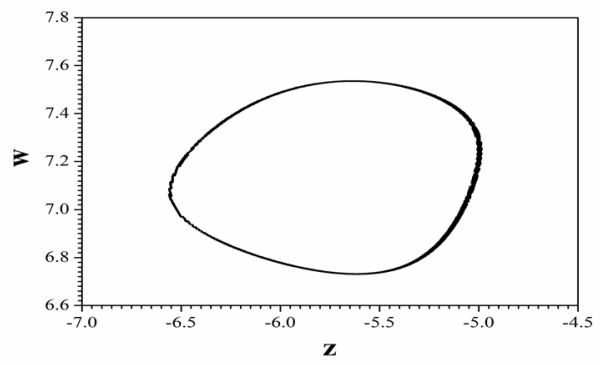

(b)

Figure 7. (a) Phase portrait and (b) Poincaré map, for $\alpha=1, b=3, c=0.1, d=6, e=1$.

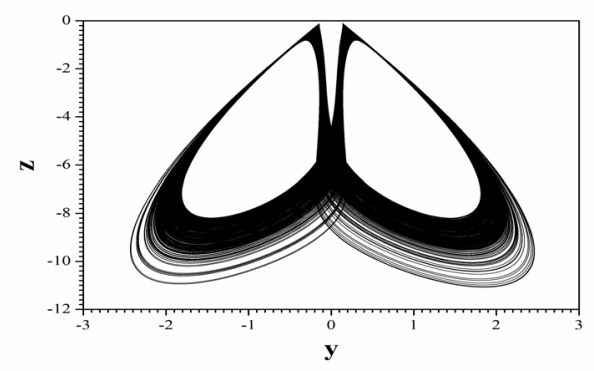

(a)

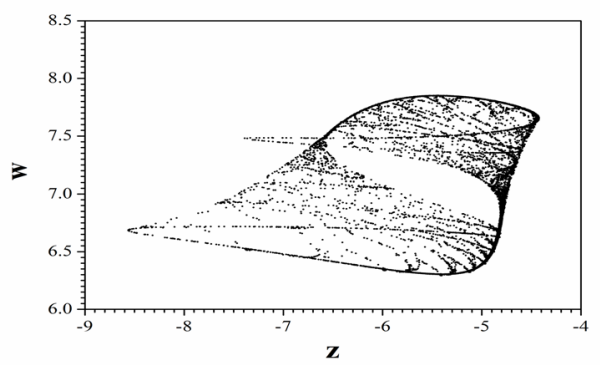

(b)

Figure 8. (a) Phase portrait and (b) Poincaré map, for $\alpha=0.85, b=3, c=0.1, d=6, e=1$.

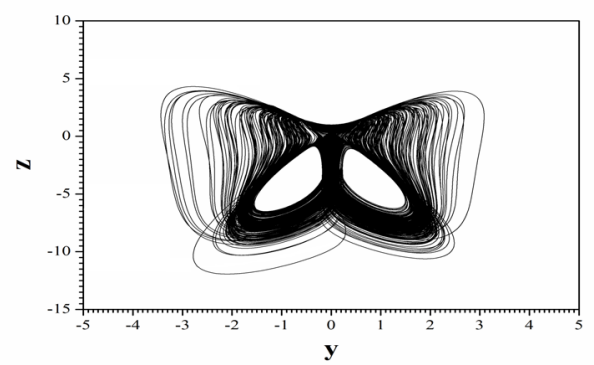

(a)

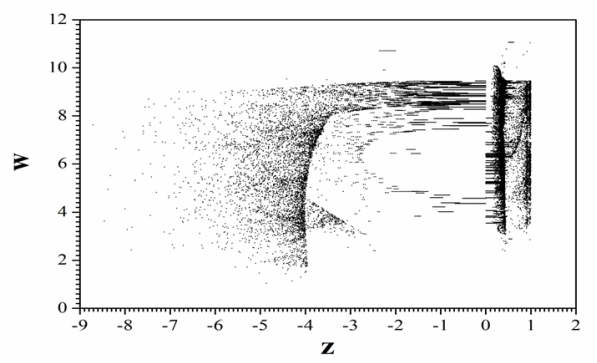

(b)

Figure 9. (a) Phase portrait and (b) Poincaré map for $\alpha=0.5, b=3, c=0.1, d=6, e=1$. 

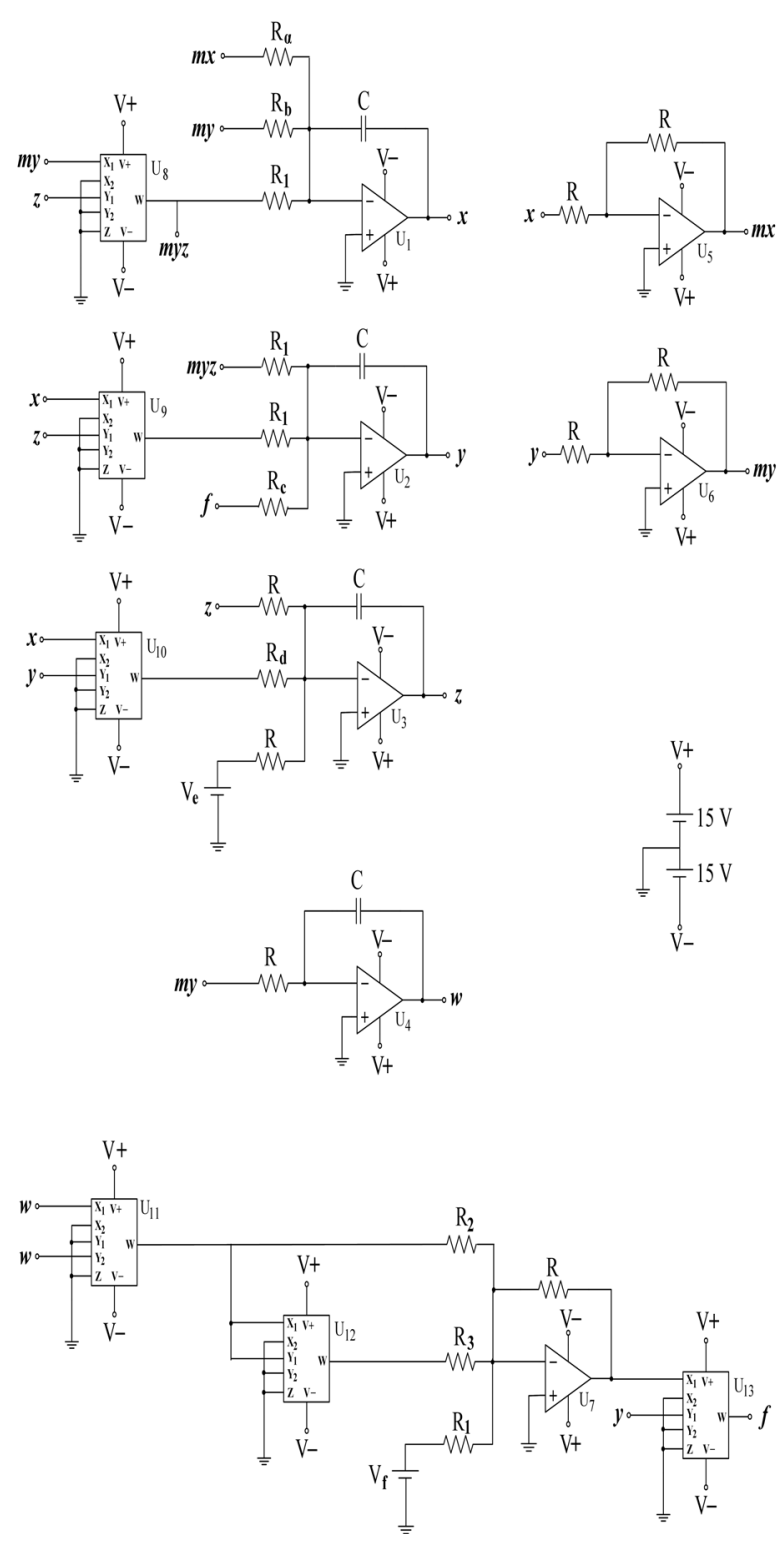

Figure 10. Schematic of the circuit which emulating the memristive hyperchaotic system.

In system (8), the variables $x, y, z$ and $\omega$ correspond to the voltages in the outputs of the integrators $U 1-U 4$. Normalizing the differential equations of system (8), by using $\tau=t / R C$, we can see that this system is equivalent to the system (6), with $a=R / R_{a}, b=R / R_{b}, c=R / R_{c}$ and $d=R /\left(10 \mathrm{~V} \cdot R_{d}\right)$. The circuit components have been selected as: $R=10 \mathrm{k} \Omega, R_{1}=1 \mathrm{k} \Omega, R_{2}=0.4 \mathrm{k} \Omega, R_{3}=0.5 \mathrm{k} \Omega$, $R_{a}=20 \mathrm{k} \Omega, R_{b}=3.333 \mathrm{k} \Omega, R_{c}=100 \mathrm{k} \Omega, R_{d}=0.166 \mathrm{k} \Omega$, $C=10 n F, V_{e}=V_{f}=-1 V_{D C}$, while the power supplies of all active devices are $15 V_{D C}$. For the chosen set of components the system's (6) parameter is $a=0.5, b=3, c=0.1$, $d=6, e=1$. The designed circuit is implemented in the electronic simulation package MULTISIM and the obtained results are displayed in Figs. 11-12. Theoretical attractors (see Fig.9a) are similar with the circuital ones (see Fig. 12).

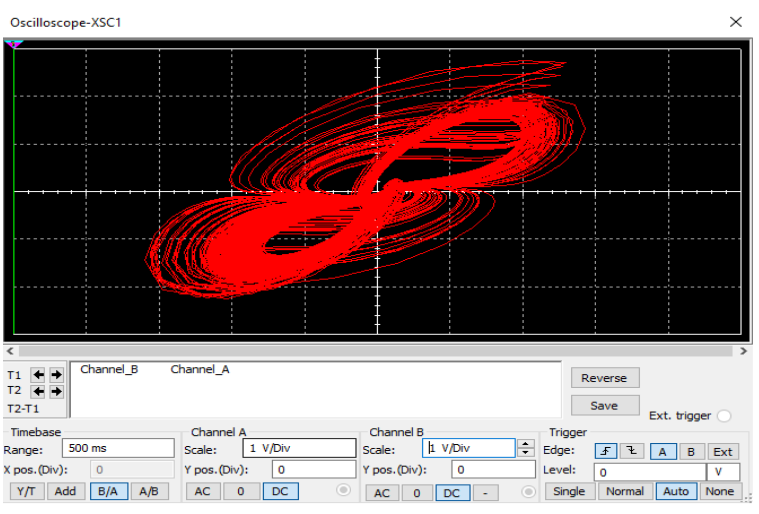

Figure 11. Hyperchaotic attractor of the designed circuit obtained from MULTISIM in the $(x, y)$-phase plane, for $a=0.5$, $b=3, c=0.1, d=6, e=1$.

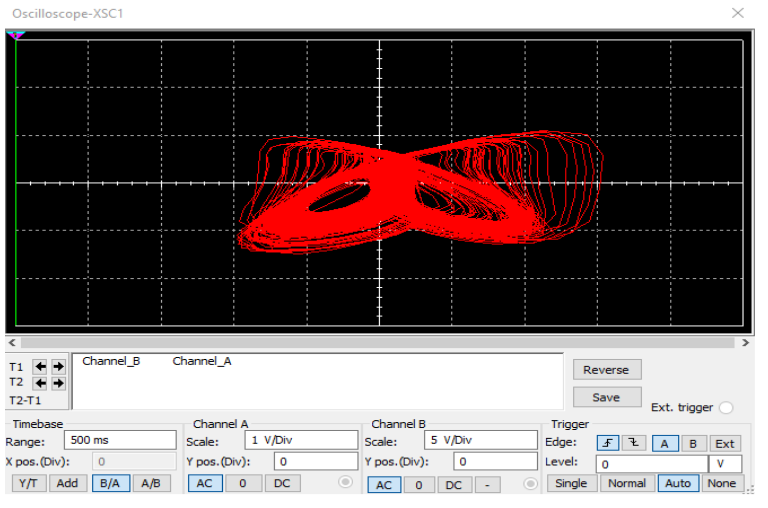

Figure 12. Hyperchaotic attractor of the designed circuit obtained from MULTISIM in the $(x, z)$-phase plane, for $a=0.5$, $b=3, c=0.1, d=6, e=1$.

\section{Conclusion}

The existence of a memristor-based hyperchaotic system with line of equibria has been studied in this paper. Although 4-D memristive systems often only generate chaos, the presence of a memristive device leads the proposed system to a hyperchaotic system with hidden attractors. The system has rich dynamical behavior as confirmed by the example of attractor reported and by the numerical Poincaré map presented. Also, the designed circuit emulates very well the proposed hyperchaotic memristive system. Because there is little knowledge about the special features of such system, future works will continue focusing on dynamical behaviors, as well as the possibility of control and synchronization of such system.

\section{References}

[1] L.O. Chua, "Memristors-the missing circuit element," IEEE Trans. Circuit Theory, vol. CT-18, pp. 507-519, (1971).

[2] L.O. Chua and S.M. Kang, "Memristive devices and systems," Proceeding of the IEEE, vol. 375, no 23, pp. 209-223, (1976). 
[3] D.B. Strukov, G.S. Snider, G.R. Stewart, and R.S. Williams, "The missing memristor found," Nature,no 453, pp. 80-83, (2008).

[4] M.D. Ventra, Y.V. Pershin, and L.O. Chua, "Circuit elements with memory: memristors, memcapacitors, and meminductors," Proceedings of the IEEE, no. 97(10), pp. 1717-1724, (2009).

[5] M. Itoh and L.O. Chua, "Memristor oscillators," Int J Bifurc Chaos, vol. 18, pp. 3183-3206, (2008).

[6] Y.B. Zhao, C.K. Tse, J.C. Feng, and Y.C. Guo, "Application of memristor-based controller for loop filter design in charge-pump phase-locked loop," Circuits Syst Signal Process, vol. 32, pp. 1013-1023, (2013).

[7] A.L. Wu, J.N. Zhang, and Z.G. Zeng, "Dynamical behaviors of a class of memristor-based Hopfield networks," Physics Letters A, vol. 375, pp. 1661-1665, (2011).

[8] C.K. Volos, I.M. Kyprianidis, and I.N. Stouboulos, "The Memristor as an Electric Synapse Synchronization Phenomena," In Proc. Int. Conf. DSP'2011 (Confu, Greece), pp. 1-6, (2011).

[9] J.J. Yang, D.B. Strukov, and D.R. Stewart, "Memristive Devices for Computing," Nature Nanotechnology, vol. 8, pp. 13-24, (2013).

[10] T. Driscoll, J. Quinn, S. Klein, H.T. Kim, B.J. Kim, Y. V. Pershin, M. Di Ventra, and D. N. Basov, "Memristive Adaptive Filters," Applied Physics Letters, vol. 97, pp. 093502-1-3, 2010.

[11] L. Wang, C. Zhang, L. Chen, J. Lai, and J. Tong, "A Novel Memristor-Based rSRAM Structure for Multiple-Bit Upsets Immunity," IEICE Electronics Express, vol. 9, pp. 861-867, (2012).

[12] Y. Shang, W. Fei, and H. Yu, "Analysis and Modeling of Internal State Variables for Dynamic Effects of Nonvolatile Memory Devices," IEEE Transactions on Circuits and Systems I: Regular Papers, vol. 59, pp. 1906-1918, (2012).

[13] S. Shin, K. Kim, and S.M. Kang, "Memristor Applications for Programmable Analog ICs," IEEE Transactions on Nanotechnology, vol. 10, pp. 266-274, (2011).

[14] C. Cepisca, N.G. Bardis., Measurement and control in street lighting (ELECTRA publication, 2011).

[15] Bogdan MARIA, Mariana BUGA, Rares MEDIANU, Costin CEPISCA, Nikolaos BARDIS, "Obtaining a Model of Photovoltaic Cell with Optimized Quantum Efficiency,'Scientific Bulletin of the Electrical Engineering Faculty, No. 2 (16), ISSN 1843-6188, (2011).

[16] Costin CEPIŞCĂ, Sorin Dan GRIGORESCU, Stergios GANATSIOS, Nikolaos G. BARDIS,"Passive and Active Compensations for Current Transformers," METROLOGIE, pp. 5-10, (4/2008)

[17] S. Jafari and J.C. Sprott, "Simple chaotic flows with a line equilibrium," Chaos, Solitons and Fractals, vol. 57, pp. 79-84, (2013).

[18] C. Li, I. Pehlivan, J.C. Sprott, and A. Agkul, "A novel four-wing strange attractor born in bistability," IEICE Electronics Express, vol.12, no 4, pp. 201111620141116, (2015).

[19] A. Wolf, J.B. Swift, H.L. Swinney, and J. Vastano, "Determining Lyapunov exponents froma timeseries," Physica D, vol. 16, no. 3, pp. 285-317, (1985).

[20] M. Barakat, A. Mansingka, Radwan, A.G., and Salama, K.N. "Generalized hardware post processing technique for chaos-based pseudorandom number generators", ETRI Journal, vol. 35, pp. 448-458, (2013).

[21] L. Gamez-Guzman, C. Cruz-Hernandez, R. LopezGutierrez, and E. E. Garcia-Guerrero, "Synchronization of Chua's circuits with multiscroll attractors: application to communication". Commun. Nonlinear Sci. Numer. Simul. , vol. 14, pp. 2765-2775, (2009).

[22] S. Sadoudi, C. Tanougast, M. S. Azzaz and A. Dandache, "Design and FPGA implementation of a wireless hyperchaotic communication system for secure realtime image transmission". EURASIP J. Image and Video Processing, vol. 943, pp. 1-18, (2013).

[23] C.K. Volos, I.M. Kyprianidis, and I.N. Stouboulos, "A chaotic path planning generator for autonomous mobile robots," Robot. Auto. Systems, vol. 60, pp. 651656, (2012).

[24] M. E. Yalcin, J.A.K. Suykens, and J. Vandewalle, "True random bit generation from a double-scroll attractor," IEEE Trans. Circuits Syst. I, Regular Papers, vol. 51, pp. 1395-1404, (2004).

[25] A. Buscarino, L. Fortuna, and M. Frasca, "Experimental robust synchronization of hyperchaotic circuits," Physica D, vol. 238, pp. 1917-1922, (2009).

[26] V. Sundarapandian and I.Pehlivan, "Analysis, control, synchronization, and circuit design of a novel chaotic system", Math. Comp. Modelling, vol. 55, pp. 1904-1915, (2012).

[27] S. Bouali, A. Buscarino, L. Fortuna, M. Frasca, and L.V. Gambuzza, "Emulating complex business cycles by using an electronic analogue". Nonl. Anal.: Real World Applications, vol. 13, pp. 2459-2465, (2012).

[28] L. Fortuna, M. Frasca, and M.G. Xibilia, "Chua's circuit implementation: Yesterday, today and tomorrow", World Scientific, Singapore, (2009). 